\title{
Effects of Glutaraldehyde on the Ryanodine Receptor by Cryo-EM
}

\author{
Joshua Strauss $^{1,2}$, Terence Wagenknecht ${ }^{1,2}$
}

1. Department of Biomedical Sciences, School of Public Health, University at Albany, State University of New York, Albany, NY, USA

2. Wadsworth Center, New York State Department of Health, Albany, NY, USA

Ryanodine receptors (RyR) are large intracellular calcium release channels that play a vital role in excitation-contraction coupling (ECC). RyR and the L-type calcium channel, also known as the dihydropyridine receptor, form core components of a large dynamic complex referred to as ECC machinery, along with several other smaller regulatory proteins including FK506 binding proteins (FKBP12 and FKBP12.6), calmodulin, and calsequestrin [1]. We have described a simple cross-linking protocol that can be used to stabilize RyR-protein ligand complexes in vitro, for characterization by single particle cryo-EM [2]. Using the skeletal FKBP12:RyR complex as a model system, we demonstrated the feasibility this method, by proof of principal. Glutaraldehyde fixation preserved the overall morphology of RyR and stabilized the FKBP12:RyR1 complex. However chemical cross-linking did induce minor changes to RyR structure at the cytoplasmic and transmembrane regions.

We present new findings showing that cross-linking with a higher concentration of glutaraldehyde seems to alter the orientational preferences of RyR1 particles when they are applied to a carbon support film (Figure 1). Methods are as described previously [2], except that the glutaraldehyde concentration was increased from 0.04 to $0.2 \%$ and the cross-linking reaction time was reduced from 20 to 10 minutes. Glutaraldehyde did not appear to alter the structure of RyR as assessed by comparison of two-dimensional averages of RyR in the fourfold symmetric orientation in the presence and absence of cross-linker (lower right Figure 1). The effect of $0.2 \%$ glutaraldehyde on particle orientation is noticeable in the micrographs, which show examples of RyR in "side view" orientations that are rarely observed for non-cross-linked receptors (Fig. 1, top two panels). Classification of the particle images into orientational classes shows a marked increase in the frequency of non-fourfold symmetric orientations (Fig. 1, bottom left). Effects of glutaraldehyde on particle orientation have been observed previously for a soluble (non-membrane) protein complex [4].

In the absence of glutaraldehyde RyR1 particles show a strongly preferred orientation in which the receptor's four-fold axis of symmetry is perpendicular to the carbon substrate. Consequently, it is necessary to tilt the specimen to obtain other orientations that are required to determine a 3D reconstruction. Glutaraldehyde cross-linking of RyRs should allow the acquisition of images of RyR1 particles in multiple orientations thereby reducing the need for tilting the specimen grid, which would greatly facilitate the determination of higher resolution reconstructions of RyR. Currently, to obtain multiple orientations of RyR it is necessary to suspend RyR in buffer over holes in a carbon support film, but this is difficult to achieve in practice, not only for RyRs, but other membrane proteins as well. 


\section{References.}

[1] Dulhunty, A, F. Clin Exp Pharmacol Physiol. 33, (2006), p. 763

[2] Strauss, J, D., and Wagenknecht, T. J Struct Biol. 181, (2013), p. 300

[3] Frank, J. et al, J Struct. Biol., 116, (1996), p. 190

[4] Kastner, B. et al, Nat Methods. 5, (2007), p. 53

[5] The authors acknowledge funding by NIH, grant \# R01 AR40615
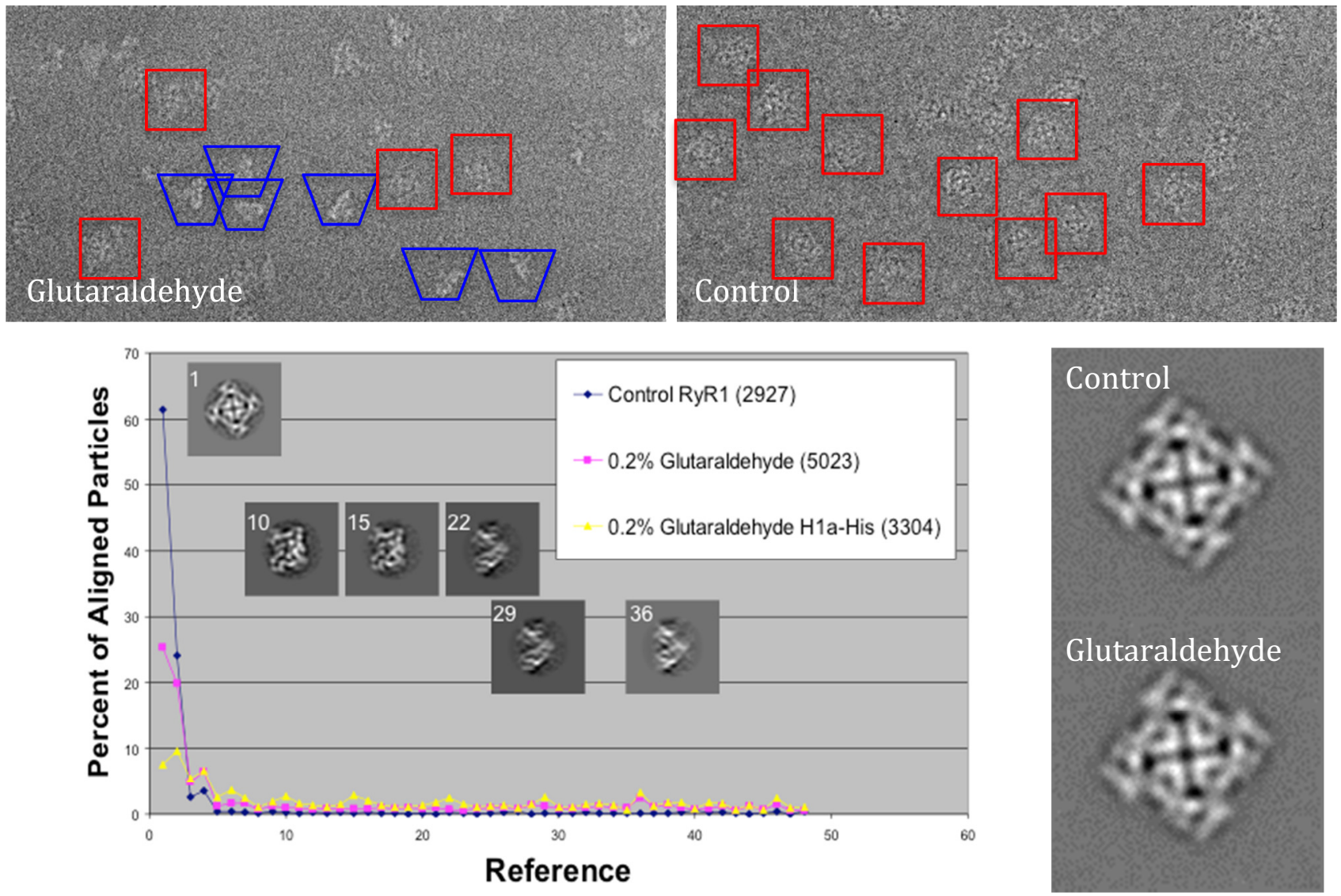

Figure 1. Selected areas of cryo-TEM micrographs showing glutaraldehyde cross-linked RyR1 particles (right image) and control RyR1 particles (left image). For the cross-linked specimen blue trapezoids indicate "side view" images that are rarely seen for non-cross-linked RyRs which invariably exhibit the square-shaped fourfold symmetric orientation. The red squares indicate fourfold symmetric views. Images were taken with a Tecnai F20 TEM at 50,000 X nominal magnification using low dose conditions (15-20 $\mathrm{e}^{2} / \AA$ ). Particles were aligned to a set of low-pass filtered RyR1 reference projections using SPIDER [3]. Lower plot shows a histogram of aligned particles with examples of RyR1 reference projections for selected orientations shown as inserts (labeled 1, 10, 15, 22,29 and 36). Cross-linked RyR1s show less preferred orientations as compared to non-crosslinked control samples. Two-dimensional averages of control RyR1 and glutaraldehyde cross-linked RyR1 (bottom right images). 